\title{
Uso do AHP na avaliação do desempenho do sistema de gestão da qualidade, meio ambiente e segurança e saúde ocupacional integrados
}

\author{
Using AHP to evaluate the performance of the quality, environment, \\ occupational health and safety management systems \\ ' Helder Antônio da Silva, ' Luiz Henrique Dias Alves, ${ }^{2}$ Fernando Augusto Silva Marins \\ 2 Messias Borges Silva y ${ }^{2}$ Ubirajara Rocha Ferreira \\ I IF SUDESTE MG - Campus Juiz de Fora (Brasil) 2UNESP - Campus Guaratinguetá (Brasil) \\ helder.silva@ifsudestemg.edu.br luiz.alves@ifsudestemg.edu.br fmarins@feg.unesp.br \\ messias.silva@feg.unesp.br ferreiur@feg.unesp.br
}

Fecha de recepción: 29-3-2010

Fecha de aceptación: 25-1-2011

Resumo: $O$ artigo analisa a aplicação do método AHP, em conjunto com o anexo A da ISO 9004:2000, para avaliar o desempenho de duas organizações industriais, certificadas pela norma ISO 900 I:2000, frente aos requisitos específicos dos sistemas de gestão da qualidade, ambiental, da gestão de segurança e saúde ocupacional. As organizações avaliadas foram uma empresa estatal do setor de ciência e tecnologia que atua na área nuclear e uma empresa do setor privado que atua na área automotiva e ferroviária. Foi utilizado o software livre Web-Hipre versão I.22 da Helsinki University of Technology da Finlândia. Os resultados apresentados demonstraram que o AHP em conjunto com o anexo A da ISO 9004:2000 é adequado para avaliar desempenho organizacional. As duas organizações apresentaram níveis de desempenho similares.

Palavras-chave: AHP, análise de desempenho, meio ambiente, qualidade, saúde ocupacional.

Abstract: The paper analyses the application of the AHP method together with the attach A of ISO 9004:2000 in order to evaluate the performance of two industrial organizations, both possessing quality management systems with ISO 900 I:2000 certifications, based on the specific requirements of quality, environment, occupational health and management systems. The evaluated organizations were a government-owned company from the science and technology sector that works in the nuclear area, and a private company that works in the automotive and railroad area. The open software Web-Hipre version 1.22 from Helsinki University of Technology, Finland, was used. The results demonstrated that the AHP method together with the attach A of ISO 9004:2000 is adequate to evaluate organizational performances. Both organizations presented similar performance levels.

Key Words: AHP, performance analysis, environment, quality, health management system

\section{Introdução}

Atualmente as organizações, principalmente as indústrias, vêm cada vez mais buscando alternativas para se manterem no mercado ou até obter novos mercados. A busca incessante pela melhoria contínua além da evolução do mercado tem exigido cada vez mais equilíbrio entre o comportamento humano e os sistemas produtivos. Algumas organizações optaram pela integração dos sistemas de gestão da qualidade, meio ambiente e segurança e saúde ocu- pacional como uma das formas ou modelos estratégicos de gestão visando à sobrevivência e melhoria frente ao mercado globalizado.

Para Wilkinson e Dale (1998), o crescente interesse nos sistemas de gestão ambiental baseado na norma ISO | 400 I, e no sistema de gestão de segurança e saúde ocupacional baseado na OHSAS I800 I, por parte das empresas que já possuem o sistema de gestão da qualidade com base na ISO 900 I implantado, levou essas empresas a buscarem na estrutura, nos 
princípios e nas características específicas dessas normas e especificação, os elementos comuns com objetivos de integração desses sistemas de gestão.

Recentemente, García-Miranda, Muñiz e Sánchez (2010) concluíram que a similaridade entre a ISO 900 I e a OHSAS I800। favorece a implantação de um Sistema de Gestão de Segurança e Saúde Ocupacional, além disso, a estrutura da ISO 900 I é semelhante e compartilha dos mesmos princípios, como os dos padrões de qualidade e meio ambiente propostos por organizações internacionais; o que torna a adoção de um Sistema Integrado de Gestão o mais viável e prático para as organizações de uma forma geral.

A Gestão Integrada alinha os Sistemas de Gestão da Qualidade (ISO 9000), Meio Ambiente (ISO I4000), Segurança e Saúde Ocupacional (OHSAS I8000) e em alguns casos inclui também a Responsabilidade Social (SA 8000). (Cerqueira, 2006; Corrêa, 2004; De Cicco, 2003; Jorgensen, 2006; Karapetrovic, 2002; Wilkinson, 1999).

Assim, no contexto competitivo, uma avaliação global faz parte das atividades de gestão de uma empresa, muito embora avaliar de maneira global e sistemática seja uma tarefa complexa. No entanto, a avaliação pode garantir o controle das atividades quer seja no nível estratégico, gerencial ou operacional de uma empresa. Uma avaliação tem também como função assegurar a tomada de decisão, fundamentada em informações e fatos de observações.

Portanto, em uma avaliação, as dificuldades encontradas iniciam-se com a definição de quais são os critérios/itens a serem examinados e que método deverá ser usado. Estes critérios são normalmente escolhidos com base na legislação acerca do assunto; já os métodos, são escolhidos por meio de observações e ensaios. Neste contexto, os critérios variam conforme a área pertinente (qualidade, ambiental, saúde e segurança). Tal variedade de critérios/itens de avaliação exige um conhecimento da inter-relação orgânica dos conceitos das três áreas (qualidade, ambiental e saúde e segurança ocupacional), até o ponto de se constituir uma unidade formada com as proposições de cada área.

Os métodos mais utlizados para realização de avaliação de desempenho e maturidade de sistemas de gestão organizacional são os métodos oriundos de prêmios da qualidade nacionais, como o Prêmio Deming, no Japão. Estes métodos são muito eficientes mas dependem de terem pessoas com experência para aplicá-los, além disso, muitas organizações que estão iniciando a implantação de sistemas de gestão se deparam com resultados um tanto quanto negativos e acabam desistindo de aplicar o método e até mesmo de implantar o sistema de gestão.

Verifica-se através de observações oriundas de pesquisas recentes, que o método AHP permite avaliar a importância de cada critério/item frente ao contexto proposto com racionalidade e precisão. Devido a estes atributos a aplicação do método AHP torna-se uma alternativa confiável para ser aplicada nesta pesquisa.

Segundo Gomes et al. (2004), um dos primeiros métodos desenvolvidos no ambiente das decisões multicritério, sendo talvez o mais usado no mundo, é o Método de Análise Hierárquica, mais conhecido como Método AHP, criado pelo Prof.Thomas L. Saaty em 1980. Nesse método, o problema de decisão é dividido em níveis hierárquicos, facilitando, assim, sua compreensão e avaliação.

Velasquez, Núñez e Monroy (2010) concluíram em um estudo realizado em uma organização do setor metal mecânico que o Método AHP permite estabelecer o nível de importância de valores organizacionais associados aos fatores que afetam a produtividade da organização, tendo um enfoque similar ao da avaliação apresentada neste trabalho.

método AHP (Analytic Hierarchy Process) tem sido empregado para situações de: definição de prioridades, avaliação de custos e benefícios, alocação de recursos, mensuração de desempenho (benchmarking), avaliação ou pesquisa de mercado, determinação de requisitos, decisões estratégicas (Forward \& Backward Planning), planejamento e seqüenciamento de atividades, previsão de cenários (forecasting), negociação e resolução de conflitos, decisões e previsões políticas ou sociais e análise de decisão sob risco. (SHIMIZU, 2006).

Forman e Selly (200I) argumentam que o nome do método AHP explica um pouco da sua lógica de aplicação:

- Analytic: Por suas características, o AHP deveria ser chamado de Processo de Síntese Hierárquica pois na sua essência o AHP auxilia na medição e sintetização de uma série de fatores envolvidos em decisões complexas; 
- Hierarchy: Subdivisão em hierarquia é uma característica que não é desconhecida às organizações. Hierarquia é a maneira adaptável para inteligência finita assumir uma situação complexa. Grandes organizações possuem estruturas hierárquicas, pois são divididas em unidades que são ainda subdivididas em outras unidades menores e assim por diante.

- Process: Um processo é uma série de ações, alterações, ou funções que nos levam a um fim ou resultado. O processo do AHP não é um modelo que descobre a resposta certa, mas um processo que ajuda os decisores a encontrar a melhor resposta.

A comparação par a par das alternativas é realizada utilizando uma escala própria, que varia de I até 9. Essa escala, denominada de Escala Fundamental, foi proposta por Saaty em 1980, juntamente com o método AHP. Segundo Luz et al. (2006), para um nível com $n$ critérios, são requeridos $n(n-1) / 2$ comparações entre $a_{i}$ e $a_{j}, \forall i, j$, construindo-se uma matriz de preferências $C_{i, j}(n \times n)$. A comparação é feita respondendo-se às questões:

- Ao influenciar o nível acima, o critério $a_{i}$ é mais importante, menos importante ou de igual importância ao critério $a_{j}, \forall i, j$;

- Para os $a_{i j}$ não equivalentes, sendo que $a_{i}$ é mais importante do que $a_{j}, a_{i}$ é: (i) um pouco mais importante do que $a_{j}$; (ii) muito mais importante do que $a_{j ;}$ (iii) muito fortemente mais importante do que $a_{j}$; ou (iv) absolutamente mais importante do que $a_{j}$.

A matriz de preferência é preenchida de acordo o Quadro I.Valores intermediários se destinam as si- tuações intermediárias, nas quais é necessário discernir mais claramente entre duas alternativas muito parecidas, segundo o critério de julgamento.

Uma vez preenchida a matriz de preferência, realizase o cálculo da importância relativa dos critérios, encontrando-se os autovetores com máximos autovalores da matriz. Os componentes do autovetor são as prioridades dos critérios e o autovetor será o valor atribuído ao nível de coerência do julgamento, dentro das bases propostas pelo método. Sendo A, a matriz de comparações, então, calcula-se o vetor de prioridade $\mathbf{w}$, tal que satisfaça a equação ( I), e a razão de coerência CR é calculada pela equação (2).

$$
\begin{gathered}
\text { A.w }=\lambda_{\max } \cdot \mathbf{w}(\mathrm{I}) \\
\mathrm{CR}=\left[\lambda_{\max }-n\right] /[\mathrm{RI}(n-\mathrm{I})]
\end{gathered}
$$

Onde: $n$ é o número de critérios; $\lambda_{\max } \leq n$ é o maior autovalor, e RI é o índice de coerência aleatório médio, extraído de Tabela de simulações com amostras de matrizes de várias dimensões. Segue o Quadro 2 com os valores de RI relacionados com n:

Quadro 2

Índice de coerência aleatório. Fonte: Salomon (2004)

\begin{tabular}{|c|c|}
\hline Ordem da matriz (n) & $\boldsymbol{R}$ \\
\hline 3 & 0,52 \\
\hline 4 & 0,89 \\
\hline 5 & 1,11 \\
\hline 6 & 1,25 \\
\hline 7 & 13,5 \\
\hline 8 & 1,40 \\
\hline 9 & 1,45 \\
\hline 10 & 1,49 \\
\hline
\end{tabular}

Quadro I

\begin{tabular}{|c|c|c|c|}
\hline se $a_{i}$ em relação a $a_{j}=$ & então $c_{i j}=$ & se $a_{i}$ em relação a $a_{j}=$ & então $c_{i j}=$ \\
\hline igual & 1 & igual & I \\
\hline um pouco mais importante & 3 & um pouco menos importante & $1 / 3$ \\
\hline muito mais importante & 5 & muito menos importante & $1 / 5$ \\
\hline fortemente mais importante & 7 & fortemente menos importante & |/7 \\
\hline absolutamente mais importante & 9 & absolutamente menos importante & 1/9 \\
\hline
\end{tabular}

Opções de preferência com base em comparações pareadas. Fonte: Saaty (|99|) 
Saaty (200 I) e Salomon (2004), retirar a vírgula recomendam que, para vetores de CR acima de 0,20, os julgamentos sejam revistos, porém, tal incoerência deve servir mais como um alerta do que um evento indesejável. Sendo assim, a revisão dos julgamentos é um procedimento sistemático de meIhoria do auxílio à decisão.

Para o presente trabalho, será utilizado o índice 0,20 para verificação da coerência dos julgamentos, conforme descrito no parágrafo anterior.

É importante examinar quão sensitiva é a alternativa preferida quanto às mudanças nos juízos emitidos pelo decisor. Sendo assim, para aplicação do método AHP neste trabalho, foi utilizado o software livre WebHipre versão I.22 da Universidade de Helsink na Finlândia, que permite realizar análise de sensibilidade dinâmica, podendo alterar os pesos dos elementos de um dado nível de hierarquia, sendo possível observar alterações nas pontuações das alternativas.

\section{Elaboração do método proposto}

\subsection{Considerações gerais}

O método consiste em uma avaliação de desempenho do sistema de gestão da qualidade, meio ambiente e da segurança e saúde ocupacional integrados, no nível estratégico, gerencial e operacional, envolvendo organizações que estejam implementando ou já tenha implementado esses sistemas integrados ou não; por meio de critérios pré estabelecidos.

Para elaboração do método proposto levou-se em consideração o levantamento bibliográfico acerca do assunto, fundamentou-se principalmente no método AHP e no método de auto avaliação do anexo A da ISO 9004:2000.

A Figura 3 apresenta esquematicamente as fases do método proposto.

\subsection{Descrição das fases do método proposto}

\subsubsection{Fase I: identificação dos itens/critérios para avalição}

A identificação dos itens/critérios é uma fase única do método, ou seja, uma vez identificado os itens/critérios pelo pesquisador, essa não mais será executa- da durante a aplicação do método proposto nas organizações. Essa identificação é realizada com base na pesquisa bibliográfica. Através da conclusão desta fase, é obtida uma estrutura hierárquica dos requisitos para avaliação da importância destes requisitos para o desempenho da organização que está sendo avaliada.

Figura 3

Fases principais do método proposto

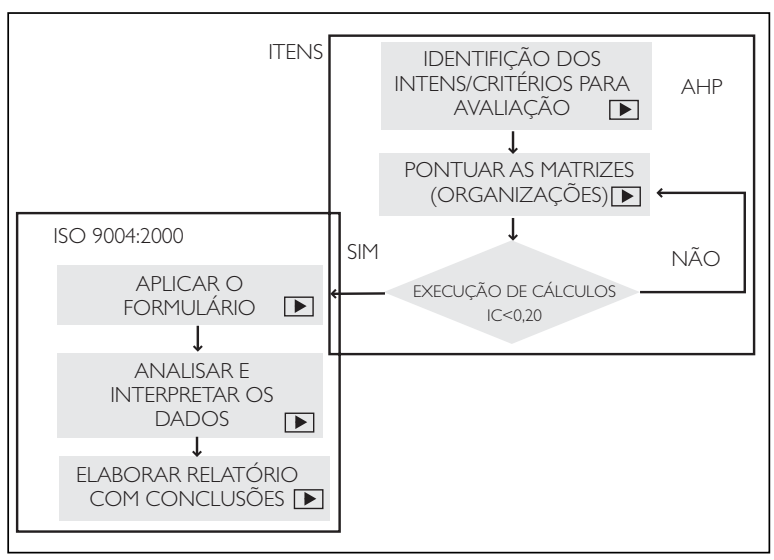

\subsubsection{Fase 2: pontuar as matrizes}

Esta fase é realizada nas organizações através das pessoas que estão envolvidas com os sistemas de gestão da qualidade, meio ambiente e da segurança e saúde ocupacional, ou ainda uma equipe que implantou ou é responsável pela manutenção do sistema integrado de gestão.

Nesta fase será realizada a avaliação paritária dos requisitos da estrutura hierárquica resultante da fase I, por meio do formulário do anexo I deste trabalho e conforme o procedimento do método AHP.Tal avaliação pode ser realizada de duas formas:

- Através de uma reunião com a equipe e o pesquisador, sendo que a pontuação a ser obtida será através de consenso das pessoas que formam a equipe; ou

- Através da distribuição para a equipe do formulário para pontuação, onde cada membro deverá avaliar e marcar no formulário o nível de importância de cada requisito. $\bigcirc$ formulário é devolvido ao pesquisador para execução dos cálculos e análises. 


\subsubsection{Fase 3: execução dos cálculos das matrizes}

Os cálculos das matrizes são executados conforme procedimento do método AHP, tendo como resultados as prioridades locais e os índices de coerência de cada matriz de julgamentos. Nos casos em que os formulários foram distribuídos, o preenchimento das matrizes com os valores de julgamento será efetuado pela média geométrica dos valores atribuídos aos formulários.

Havendo alguma matriz com índice de coerência maior que 0,20 , deverão ser avaliadas a necessidade de se refazer o julgamento para esta matriz e refazer os cálculos das prioridades locais desta matriz.

\subsubsection{Fase 4: aplicar o formulário para levantamento do desempenho}

Nesta fase será aplicado nas organizações o formulário para levantamento do desempenho da organização, podendo ser realizada de duas formas:

- Através de reunião com a mesma equipe que participou da fase 2, sendo o resultado obtido por meio de consenso dos membros da equipe; ou

- Através da distribuição do formulário para levantamento do desempenho para a equipe, onde cada membro deverá avaliar e marcar no formulário o nível de desempenho em que a organização se encontra em cada requisito. $\bigcirc$ formulário é devolvido ao pesquisador para execução dos cálculos e análises.

\subsubsection{Fase 5: análise e interpretação dos dados}

A análise dos dados será realizada mediante a obtenção dos resultados dos dois formulários, sendo efetuado o produto dos valores das prioridades locais de cada requisito, obtidos por meio do método AHP, pelos valores percentuais obtidos por meio do formulário de levantamento do desempenho. Serão analisados então os valores de desempenhos totais, obtidos por meio do somatório dos produtos, conforme procedimento descrito acima, de cada organização, comparando e interpretando estes valores e os valores de cada requisito.

\subsubsection{Fase 6: elaboração do relatório de conclusões}

Nesta fase, será apresentada pelo pesquisador a síntese dos resultados contendo o percentual global de desempenho obtido por cada organização avaliada e os valores obtidos para cada requisito avaliado nestas organizações. Estes resultados serão acompanhados de comentários acerca das constatações de pontos fortes e oportunidades de melhorias para os requisitos que mais se destacaram na avaliação, sendo este um indicativo de onde a organização deve se esforçar para melhorar seu desempenho global.

\subsection{Estrutura hierárquica dos requisitos}

A estrutura hierárquica dos requisitos é obtida na fase I do método proposto e tem como objetivo principal promover uma distribuição dos requisitos em critérios e subcritérios, criando assim uma hierarquia para avaliar a importância de cada requisito para o desempenho da organização.

Sendo assim, a Figura 4 apresenta a estrutura hierárquica para avaliação do desempenho das organizações que serão avaliadas neste trabalho.

O objetivo, indicado na caixa superior da estrutura hierárquica representa a questão que se deseja resolver, sendo em geral preenchida com uma pergunta. Para o presente trabalho a questão que se deseja conhecer ou resolver é relativo ao desempenho de organizações. Portanto a pergunta foi formulada no sentido de se conhecer qual dos requisitos ou itens possui maior importância para o desempenho de uma organização.

Os critérios são indicados nas caixas logo abaixo do objetivo. Para o presente trabalho os critérios são os requisitos de nível mais elevados baseados nos sistemas de gestão da qualidade, meio ambiente e da segurança e saúde ocupacional, suas respectivas normas, prêmios e métodos de avaliações específicos, além do método de avaliação do anexo A da ISO 9004:2000.

Os subcritérios são os desdobramentos dos critérios, são indicados pelas caixas abaixo dos critérios. Da mesma forma que os critérios, os subcritérios são também baseados nos sistemas de gestão da qualidade, meio ambiente e da segurança e saúde ocupacional, suas respectivas normas, prêmios e métodos de avaliações específicos, além do método de avaliação do anexo A da ISO 9004:2000.

\section{Aplicação do método proposto}

\subsection{Considerações gerais}

A necessidade de se avaliar organizações por meio de um método simples e flexível tem sido o foco 
Figura 4

Estrutura Hierárquica dos Requisitos

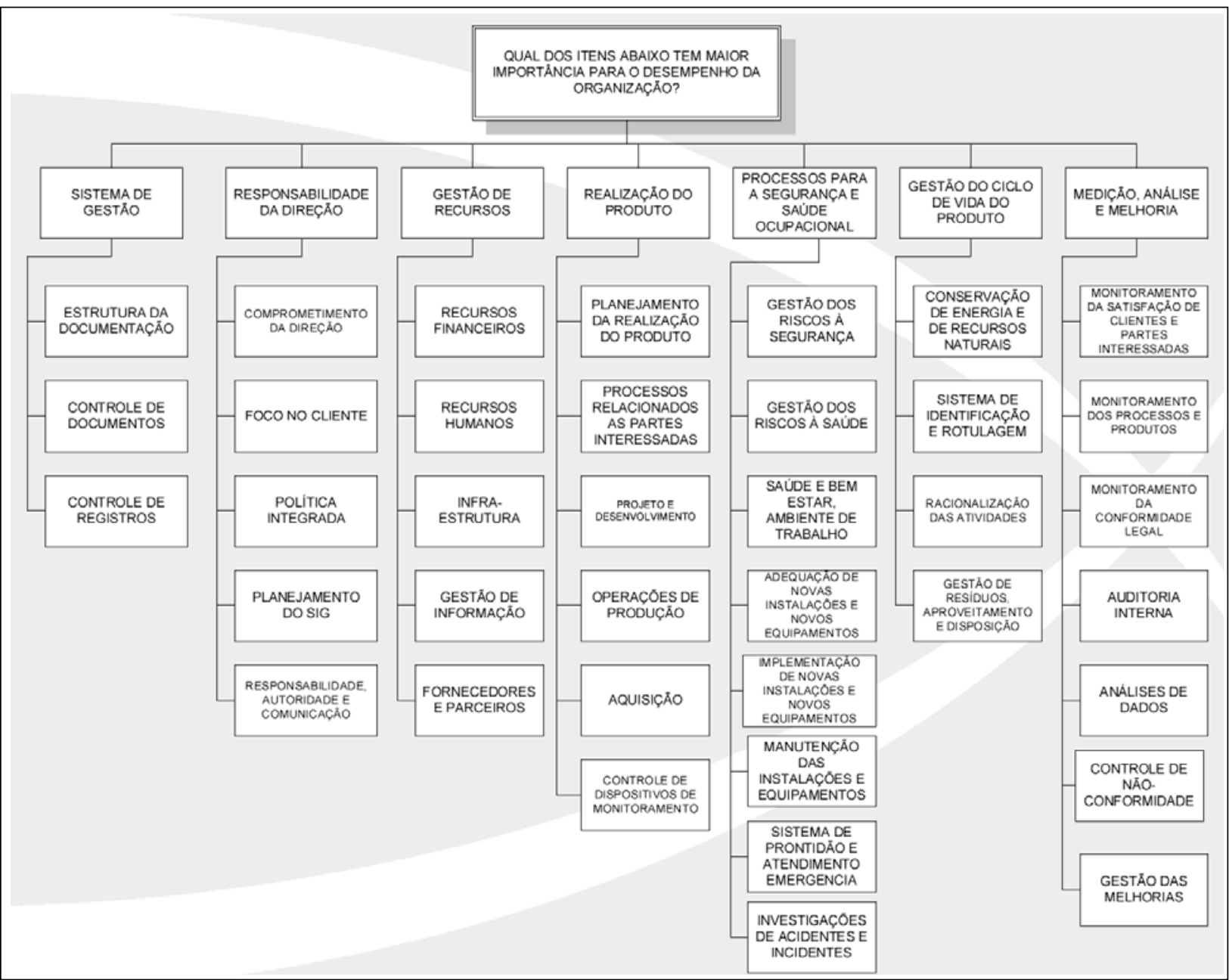

principal do desenvolvimento deste trabalho, no contexto do sistema integrado de gestão.

Portanto, neste contexto, a proposta de um método de avaliação baseada no método AHP e no método de auto-avaliação do anexo A da ISO 9004:2000, propondo critérios amparados e estabelecidos mediante uma fundamentação teórica e um sistema de medição no qual se avalia a importância e o comportamento das organizações face à esses critérios, é o que se buscou realizar neste trabalho.

Sendo assim, uma aplicação do método proposto em organizações que possuem necessidades semelhantes em relação aos sistemas de gestão integrado, devido à busca da manutenção e da melhoria de sua atuação no cenário empresarial nacional e internacional, se faz necessário no sentido de obter dados reais para comprovação da eficácia desse método.
É importante frisar a validade do método para potencializar a sua aplicação para diagnóstico do desempenho da organização, contribuindo para o processo de tomada de decisão, tendo como objetivo a melhoria contínua dos seus processos e do sistema de gestão implantado, sendo este sistema integrado ou não.

\section{Resultados e discusões}

Este item busca apresentar os resultados obtidos da aplicação do método proposto em cada organização. Para isso, será necessário identificar as organizações de forma fácil e sem quebrar o sigilo acordado. Sendo assim, chamaremos as organizações por Organização A e Organização B.

O que diferencia uma organização da outra é o tempo que cada organização possui de implantação do sistema de gestão integrado. 


\section{I. Organização A}

Esta organização possui um sistema de gestão integrado há um ano. Inicialmente esta organização havia implantado o sistema de gestão da qualidade com base na norma ISO 900I, tendo dez anos de implantação.

A aplicação do método na Organização A iniciou-se com uma reunião entre o pesquisador e os membros das coordenações da gestão da qualidade, da gestão ambiental e da gestão de segurança e saúde ocupacional no site que foi implantado esses sistemas. Nesta reunião foi explicado o método proposto e em seguida foi executada a pontuação das matrizes de julgamentos, sendo um total de oito matrizes. A Tabelal apresenta os valores obtidos do julgamento da matriz principal. Procurou-se sempre a obtenção do consenso entre os participantes para o preenchimento destas matrizes.

Na Figura 5 observa-se por meio da análise de sensibilidade que para empresa estatal, o critério/requisito realização do produto foi considerado o mais importante com índice 0,20, tendo como subcritério mais importante operação de produção, com ín- dice de 0,048 o que representa a realidade desta empresa. Se o índice do critério/requisito for acrescido de 0,03 , o subcritério mais importante será projeto e desenvolvimento.

A aplicação do formulário de avaliação do nível de desempenho dos critérios, anexo I, se deu por meio de entrevistas com os mesmos membros da equipe que participou do processo inicial da aplicação do método proposto. Os valores obtidos em cada pontuação foi registrado e o resultado final foi obtido por meio do cálculo da média aritmética dos valores registrados.

A Tabela 2 apresenta os valores obtidos na avaliação da Organização A para os critérios, subcritérios e o desempenho geral desta organização. Os valores da coluna desempenho foram obtidos através do produto dos valores da coluna de importância e dos valores da coluna maturidade para cada subcritério. Por meio do somatório dos valores da coluna desempenho de cada subcritério, obtém-se os valores de desempenho de cada critério. $O$ desempenho geral é obtido pelo somatório dos valores da coluna desempenho dos critérios.

TABELA I

Matriz Principal de Julgamentos da Organização A

\begin{tabular}{|c|c|c|c|c|c|c|c|c|}
\hline & $\begin{array}{c}\text { SISTEMA } \\
\text { DE GESTÃO }\end{array}$ & $\begin{array}{l}\text { RESPONSABILI- } \\
\text { DADE DA } \\
\text { DIREÇÃO }\end{array}$ & $\begin{array}{c}\text { GESTÃO } \\
\text { DE RECURSOS }\end{array}$ & $\begin{array}{l}\text { REALIZAÇÃO } \\
\text { DO PRODUTO }\end{array}$ & \begin{tabular}{|} 
PROCESSOS \\
PARAA \\
SEGURANÇA \\
E SAÚDE \\
OCUPACIONAL
\end{tabular} & $\begin{array}{l}\text { GESTÃO DO } \\
\text { CICLO DEVIDA } \\
\text { DO PRODUTO }\end{array}$ & $\begin{array}{l}\text { MEDIÇÃO, } \\
\text { ANÁLISE } \\
\text { E MELHORIA }\end{array}$ & $\begin{array}{c}\text { PRIORIDADE } \\
\text { RELATIVA }\end{array}$ \\
\hline $\begin{array}{l}\text { SISTEMA DE } \\
\text { GESTÃO }\end{array}$ & I & 1 & I & $1 / 2$ & 2 & $1 / 2$ & 1 & 0,126 \\
\hline $\begin{array}{l}\text { RESPONSA- } \\
\text { BILIDADE } \\
\text { DA DIREÇÃO }\end{array}$ & 1 & 1 & 2 & I & 2 & I & I & 0,166 \\
\hline $\begin{array}{c}\text { GESTÃO } \\
\text { DE RECURSOS }\end{array}$ & 1 & $1 / 2$ & 1 & $1 / 2$ & 1 & $1 / 2$ & 2 & 0,118 \\
\hline $\begin{array}{l}\text { REALIZAÇÃO } \\
\text { DO PRODUTO }\end{array}$ & 2 & I & 2 & 1 & 2 & 2 & I & 0,204 \\
\hline $\begin{array}{l}\text { PROCESSOS } \\
\text { PARAA } \\
\text { SEGURANÇA } \\
\text { ESAÚDE } \\
\text { OCUPACIONAL }\end{array}$ & $1 / 2$ & $1 / 2$ & I & $1 / 2$ & 1 & I & 1 & 0,102 \\
\hline $\begin{array}{c}\text { GESTÃO } \\
\text { DO CICLO DE } \\
\text { VIDA DO } \\
\text { PRODUTO }\end{array}$ & 2 & 1 & 2 & $1 / 2$ & I & 1 & I & 0,155 \\
\hline $\begin{array}{l}\text { MEDIÇÃO, } \\
\text { ANÁLISE } \\
\text { E MELHORIA }\end{array}$ & 1 & 1 & $1 / 2$ & I & 1 & 1 & 1 & 0,128 \\
\hline
\end{tabular}


Figura 5

Análise de sensibilidade da Organização A. Fonte: Software Web-Hipre versão I.22

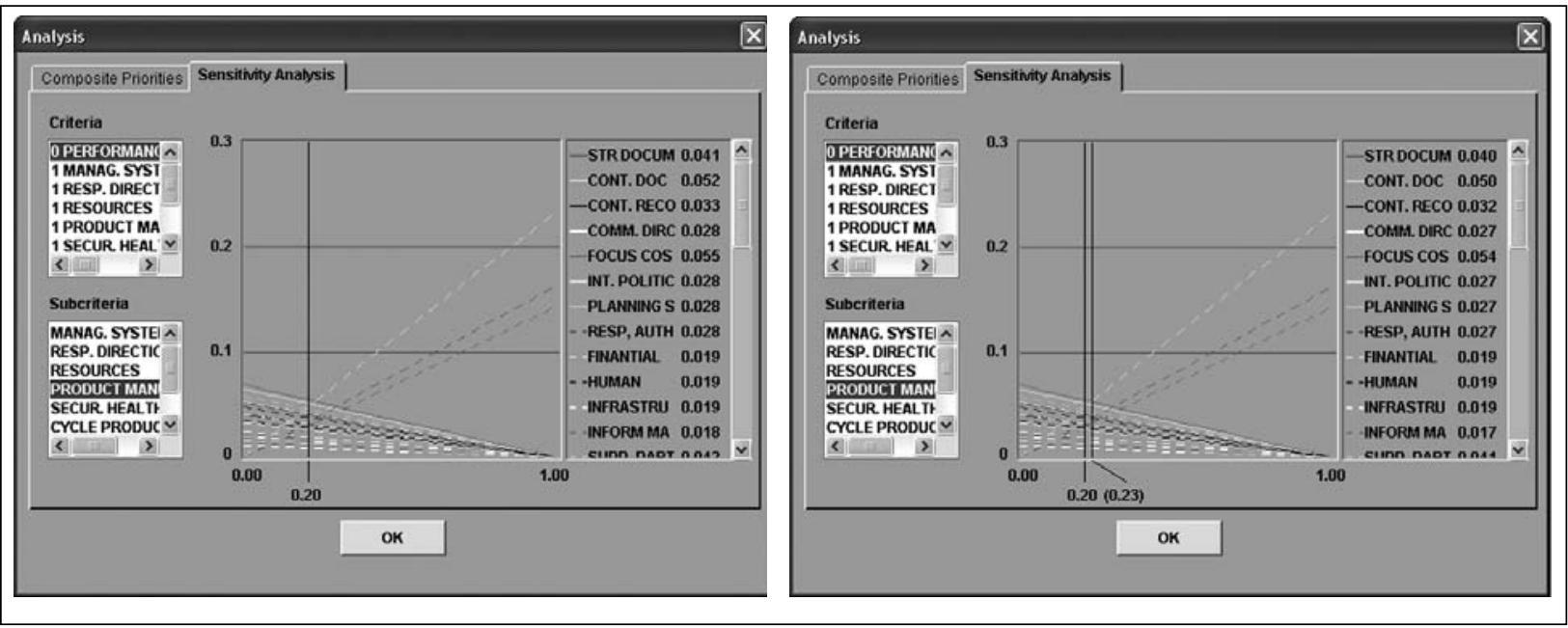

TABELA 2

Relsultado do desempenho da Organização A

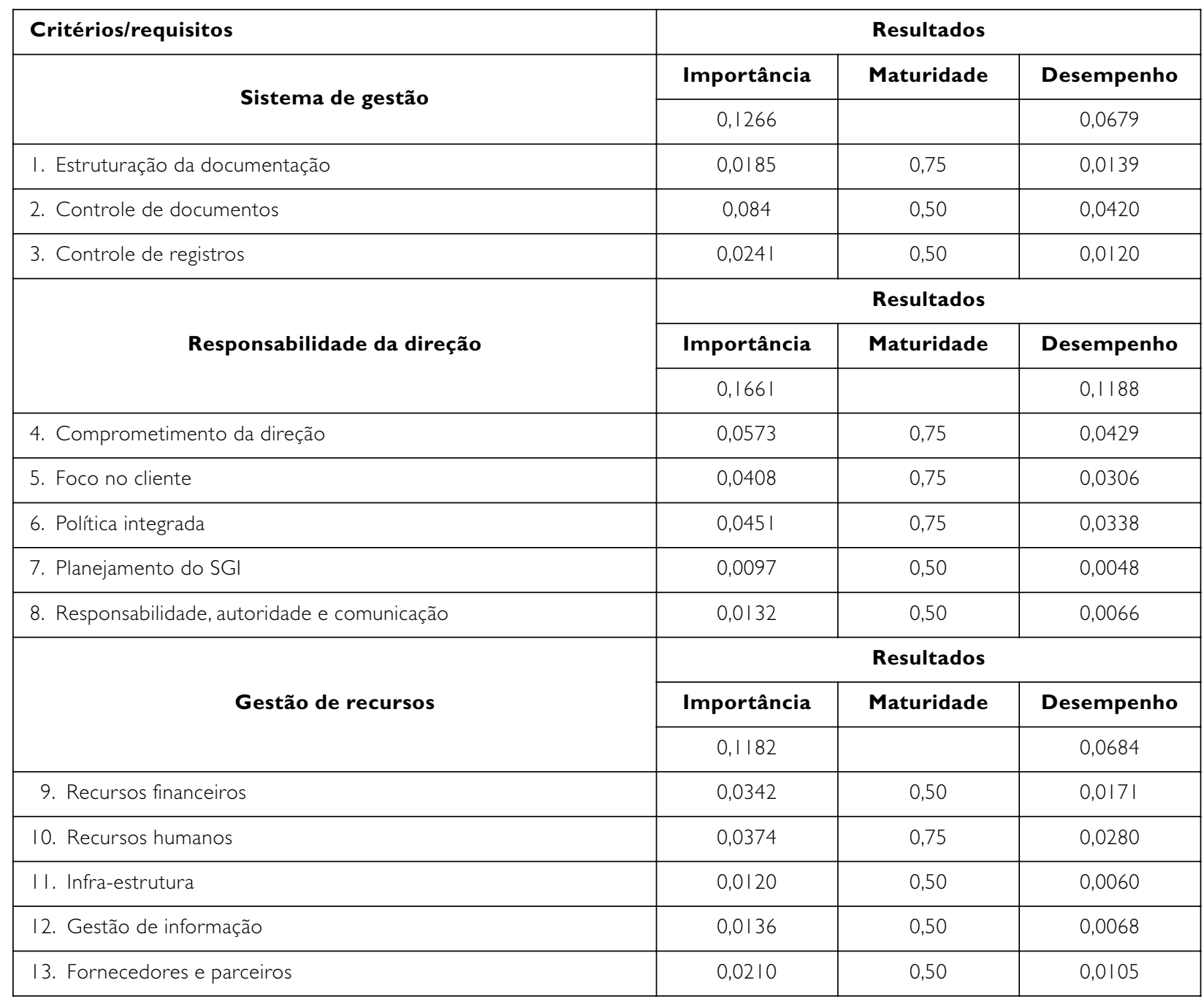


TABELA 2

Relsultado do desempenho da Organização A (continuación)

\begin{tabular}{|c|c|c|c|}
\hline \multirow{3}{*}{ Realização do produto } & \multicolumn{3}{|c|}{ Resultados } \\
\hline & Importância & Maturidade & Desempenho \\
\hline & 0,2033 & & 0,1399 \\
\hline 14. Planejamento da realização do produto & 0,0362 & 0,75 & $0,027 \mid$ \\
\hline 15. Processos relacionados as partes interessadas & 0,0386 & 0,50 & 0,0193 \\
\hline 16. Projeto e desenvolvimento & 0,0477 & 0,75 & 0,0358 \\
\hline 17. Operações de produção & 0,0484 & 0,75 & 0,0363 \\
\hline 18. Aquisição & 0,0115 & 0,50 & 0,0057 \\
\hline 19. Controle de dispositivos de monitoramento & 0,0209 & 0,75 & 0,0157 \\
\hline \multirow{3}{*}{ Processos para a segurança e saúde ocupacional } & \multicolumn{3}{|c|}{ Resultados } \\
\hline & Importância & Maturidade & Desempenho \\
\hline & 0,1022 & & 0,0655 \\
\hline 20. Gestão dos riscos à segurança & 0,0203 & 0,75 & 0,0152 \\
\hline 21. Gestão dos riscos à saúde & 0,0148 & 0,75 & 0,0111 \\
\hline 22. Saúde e bem estar, ambiente de trabalho & 0,0153 & 0,50 & 0,0076 \\
\hline 23 Adequação às inovações dos processos & 0,0108 & 0,50 & 0,0054 \\
\hline 24. Implementação de novas instalações e novos equipamentos & 0,0087 & 0,75 & 0,0065 \\
\hline 25. Manutenção das instalações e equipamentos & 0,0062 & 0,50 & 0,0031 \\
\hline 26. Sistema de prontidão e atendimento à emergência & 0,0138 & 0,75 & 0,0103 \\
\hline 27. Investigações de acidentes e incidentes & 0,0123 & 0,50 & 0,0061 \\
\hline \multirow{3}{*}{ Gestão do ciclo de vida do produto } & \multicolumn{3}{|c|}{ Resultados } \\
\hline & Importância & Maturidade & Desempenho \\
\hline & 0,1543 & & 0,0862 \\
\hline 28. Conservação de energia e recursos naturais & 0,0487 & 0,50 & 0,0243 \\
\hline 29. Sistema de identificação e rotulagem & 0,0258 & 0,25 & 0,0064 \\
\hline 30. Racionalização das atividades & 0,0177 & 0,50 & 0,0088 \\
\hline 31. Gestão de resíduos, aproveitamento e disposição & 0,0621 & 0,75 & 0,0465 \\
\hline \multirow{3}{*}{ Medição, análise e melhoria } & \multicolumn{3}{|c|}{ Resultados } \\
\hline & Importância & Maturidade & Desempenho \\
\hline & 0,1293 & & 0,0839 \\
\hline 32. Monitoramento da satisfação de clientes e partes interessadas & 0,0185 & 0,75 & 0,0139 \\
\hline 33. Monitoramento dos processos e produtos & 0,0116 & 0,75 & 0,0087 \\
\hline 34. Monitoramento da conformidade legal & 0,0203 & 0,75 & 0,0152 \\
\hline 35. Auditoria interna & 0,0358 & 0,50 & 0,0179 \\
\hline 36. Análise de dados & 0,0068 & 0,50 & 0,0034 \\
\hline 37. Controle de não-conformidade & 0,0267 & 0,75 & 0,0200 \\
\hline 38. Gestão das melhorias & 0,0096 & 0,50 & 0,0048 \\
\hline Desempenho geral da organização A & \multicolumn{3}{|c|}{$60,03 \%$} \\
\hline
\end{tabular}


Observou-se que para esta organização o critério mais importante para o seu desempenho é a realização do produto, porém o seu nível de desempenho pode ser melhorado.Tal fato é devido à implantação do sistema de gestão da qualidade ter sido implementado há dez anos.

Dentro do critério de realização do produto a Organização A destaca como sendo mais importante para o seu desempenho o subcritério operações de produção, obtendo um nível de desempenho maior dentre os outros subcritérios avaliados.

critério processos para a segurança e saúde ocupacional apresentou o menor índice nesta avaliação de desempenho, seguido do critério de gestão de recursos. Tal fato é, segundo a equipe responsável pelo sistema de gestão integrado, devido ao pouco tempo de implantação do sistema de gestão de segurança e saúde ocupacional e sua integração com os sistemas de gestão da qualidade e ambiental.

desempenho geral da Organização A foi de $60,03 \%$, sendo um valor 10\% acima da média da escala de desempenho. Este desempenho reflete o início de melhoria do desempenho da organização, que deverá se esforçar para melhorar os critérios que apresentaram valores baixos.

\subsection{Organização $B$}

Esta organização ainda não possui um sistema de gestão integrado. Inicialmente esta organização implantou o sistema de gestão da qualidade com base na norma ISO 900I, tendo mais de dez anos de implantação. A aplicação do método na Organização B iniciou-se com uma reunião entre o pesquisador e os membros das coordenações da gestão da qualidade, da gestão ambiental e da gestão de segurança e saúde ocupacional no site que foi implantado esses sistemas. Nesta reunião foi explicado o método proposto e em seguida foi executada a pontuação das matrizes de julgamento, sendo um total de oito matrizes. A Tabela 3 apresenta os valores obtidos do julgamento da matriz principal. Procurou-se sempre a obtenção do consenso entre os participantes para o preenchimento destas matrizes.

A aplicação do formulário de avaliação do nível de desempenho dos critérios, se deu por meio da distribuição do formulário para os mesmos membros

TABELA 3

Matriz Principal de Julgamentos da Organização B

\begin{tabular}{|c|c|c|c|c|c|c|c|c|}
\hline & $\begin{array}{c}\text { SISTEMA } \\
\text { DE GESTÃO }\end{array}$ & $\begin{array}{c}\text { RESPONSABILI- } \\
\text { DADE DA } \\
\text { DIREÇÃO }\end{array}$ & $\begin{array}{c}\text { GESTÃO } \\
\text { DE RECURSOS }\end{array}$ & $\begin{array}{l}\text { REALIZAÇÃO } \\
\text { DO PRODUTO }\end{array}$ & $\begin{array}{l}\text { PROCESSOS } \\
\text { PARAA } \\
\text { SEGURANÇA } \\
\text { E SAÚDE } \\
\text { OCUPACIONAL }\end{array}$ & $\begin{array}{l}\text { GESTÃO DO } \\
\text { CICLO DEVIDA } \\
\text { DO PRODUTO }\end{array}$ & $\begin{array}{l}\text { MEDIÇÃO, } \\
\text { ANÁLISE } \\
\text { E MELHORIA }\end{array}$ & $\begin{array}{l}\text { PRIORIDADE } \\
\text { RELATIVA }\end{array}$ \\
\hline $\begin{array}{l}\text { SISTEMA DE } \\
\text { GESTÃO }\end{array}$ & I & 3 & 3 & 2 & 3 & 3 & 3 & 0,310 \\
\hline $\begin{array}{l}\text { RESPONSA- } \\
\text { BILIDADE } \\
\text { DA DIREÇÃO }\end{array}$ & $1 / 3$ & 1 & $1 / 2$ & $1 / 2$ & 1 & 2 & I & 0,098 \\
\hline $\begin{array}{c}\text { GESTÃO } \\
\text { DE RECURSOS }\end{array}$ & $1 / 3$ & 2 & 1 & I & 2 & 2 & 2 & 0,161 \\
\hline $\begin{array}{l}\text { REALIZAÇÃO } \\
\text { DO PRODUTO }\end{array}$ & $1 / 2$ & 2 & I & 1 & 2 & 3 & 2 & 0,179 \\
\hline $\begin{array}{l}\text { PROCESSOS } \\
\text { PARAA } \\
\text { SEGURANÇA } \\
\text { ESAÚDE } \\
\text { OCUPACIONAL }\end{array}$ & $1 / 3$ & I & $1 / 2$ & $1 / 2$ & I & I & I & 0,088 \\
\hline $\begin{array}{c}\text { GESTÃO } \\
\text { DO CICLO DE } \\
\text { VIDA DO } \\
\text { PRODUTO }\end{array}$ & $1 / 3$ & $1 / 2$ & $1 / 2$ & $1 / 3$ & I & 1 & I & 0,077 \\
\hline $\begin{array}{l}\text { MEDIÇÃO, } \\
\text { ANÁLISE } \\
\text { E MELHORIA }\end{array}$ & $1 / 3$ & 1 & $1 / 2$ & $1 / 2$ & 1 & 1 & 1 & 0,088 \\
\hline
\end{tabular}


Figura 6

Análise de sensibilidade da Organização B. Fonte: Software Web-Hipre versão I.22.

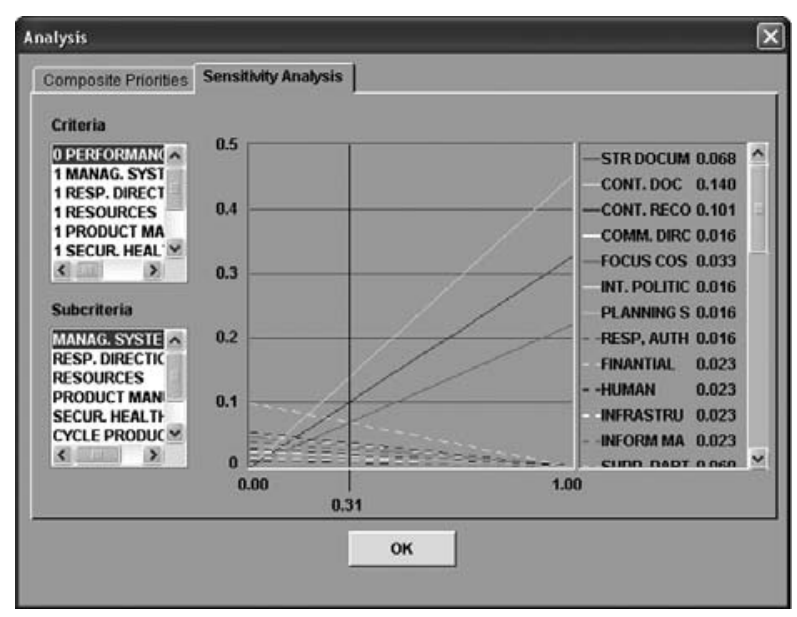

da equipe que participou do processo inicial da aplicação do método proposto. Os valores obtidos em cada pontuação foi registrado no formulário do anexo I, e o resultado final foi obtido por meio do cálculo da média aritmética dos valores registrados.

A Tabela 4 apresenta os valores obtidos na avaliação da Organização B para os critérios, subcritérios e o desempenho geral desta organização. Os valores da coluna desempenho foram obtidos através do pro-

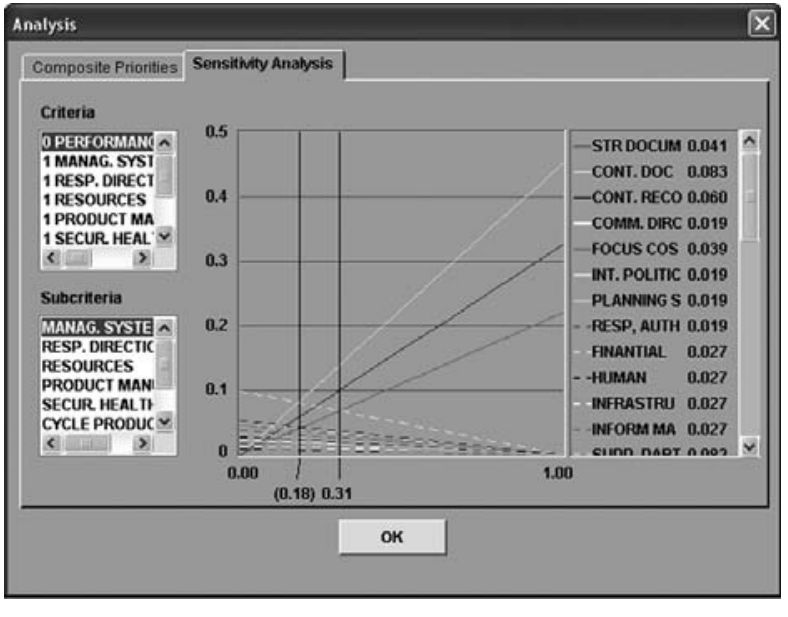

duto dos valores da coluna de importância e dos valores da coluna maturidade para cada subcritério. Por meio do somatório dos valores da coluna desempenho de cada subcritério, obtem-se os valores de desempenho de cada critério. $\bigcirc$ desempenho geral é obtido pelo somatório dos valores da coluna desempenho dos critérios.

TABELA 4

Relsultado do desempenho da Organização B

\begin{tabular}{|c|c|c|c|}
\hline Critérios/requisitos & \multicolumn{3}{|c|}{ Resultados } \\
\hline \multirow{2}{*}{ Sistema de gestão } & Importância & Maturidade & Desempenho \\
\hline & 0,317 & & 0,2377 \\
\hline I. Estruturação da documentação & 0,052 & 0,75 & 0,0390 \\
\hline 2. Controle de documentos & 0,136 & 0,75 & 0,1022 \\
\hline 3. Controle de registros & 0,129 & 0,75 & 0,0967 \\
\hline \multirow{3}{*}{ Responsabilidade da direção } & \multicolumn{3}{|c|}{ Resultados } \\
\hline & Importância & Maturidade & Desempenho \\
\hline & 0,092 & & 0,03634 \\
\hline 4. Comprometimento da direção & 0,022 & 0,50 & 0,010806 \\
\hline 5. Foco no cliente & 0,022 & 0,50 & 0,010806 \\
\hline 6. Política integrada & 0,026 & 0,25 & 0,006574 \\
\hline 7. Planejamento do SGI & 0,013 & 0,25 & 0,003287 \\
\hline 8. Responsabilidade, autoridade e comunicação & 0,010 & 0,50 & 0,004866 \\
\hline
\end{tabular}


TABELA 4

Relsultado do desempenho da Organização B (continuación)

\begin{tabular}{|c|c|c|c|}
\hline Critérios/requisitos & \multicolumn{3}{|c|}{ Resultados } \\
\hline \multirow{2}{*}{ Gestão de recursos } & Importância & Maturidade & Desempenho \\
\hline & 0,184 & & 0,113439 \\
\hline 9. Recursos financeiros & 0,022 & 0,75 & 0,059308 \\
\hline 10. Recursos humanos & 0,022 & 0,50 & 0,023552 \\
\hline II. Infra-estrutura & 0,026 & 0,50 & 0,007687 \\
\hline 12. Gestão de informação & 0,013 & 0,75 & 0,018275 \\
\hline 13. Fornecedores e parceiros & 0,010 & 0,25 & 0,004617 \\
\hline \multirow{3}{*}{ Realização do produto } & \multicolumn{3}{|c|}{ Resultados } \\
\hline & Importância & Maturidade & Desempenho \\
\hline & $0,17 \mid 427$ & & 0,112122 \\
\hline 14. Planejamento da realização do produto & 0,024608 & 0,75 & 0,018456 \\
\hline 15. Processos relacionados as partes interessadas & 0,037234 & 0,75 & 0,027925 \\
\hline 16. Projeto e desenvolvimento & 0,031004 & 0,75 & 0,023253 \\
\hline 17. Operações de produção & 0,046912 & 0,75 & $0,035 \mid 84$ \\
\hline 18. Aquisição & 0,014608 & 0,50 & 0,007304 \\
\hline 19. Controle de dispositivos de monitoramento & 0,017062 & 0,75 & 0,012797 \\
\hline \multirow{3}{*}{ Processos para a segurança e saúde ocupacional } & \multicolumn{3}{|c|}{ Resultados } \\
\hline & Importância & Maturidade & Desempenho \\
\hline & 0,09539 & & 0,033540 \\
\hline 20. Gestão dos riscos à segurança & 0,01648 & 0,50 & 0,008241 \\
\hline 21. Gestão dos riscos à saúde & 0,00761 & 0,50 & 0,003803 \\
\hline 22. Saúde e bem estar, ambiente de trabalho & 0,00663 & 0,25 & 0,001658 \\
\hline 23 Adequação às inovações dos processos & 0,00873 & 0,25 & 0,002182 \\
\hline 24. Implementação de novas instalações e novos equipamentos & 0,01001 & 0,25 & 0,002503 \\
\hline 25. Manutenção das instalações e equipamentos & 0,00761 & 0,25 & 0,001902 \\
\hline 26. Sistema de prontidão e atendimento à emergência & 0,02366 & 0,25 & $0,0059 \mid 4$ \\
\hline 27. Investigações de acidentes e incidentes & $0,0 \mid 468$ & 0,50 & 0,007338 \\
\hline \multirow{3}{*}{ Gestão do ciclo de vida do produto } & \multicolumn{3}{|c|}{ Resultados } \\
\hline & Importância & Maturidade & Desempenho \\
\hline & 0,061 & & 0 \\
\hline 28. Conservação de energia e recursos naturais & 0,010 & 0 & 0 \\
\hline 29. Sistema de identificação e rotulagem & 0,024 & 0 & 0 \\
\hline 30. Racionalização das atividades & 0,010 & 0 & 0 \\
\hline 31. Gestão de resíduos, aproveitamento e disposição & 0,017 & 0 & 0 \\
\hline
\end{tabular}


TABELA 4

Relsultado do desempenho da Organização B (continuación)

\begin{tabular}{|l|c|c|c|}
\hline \multicolumn{1}{|c|}{ Medição, análise e melhoria } & \multicolumn{2}{|c|}{ Resultados } \\
\hline & Importância & Maturidade & Desempenho \\
\cline { 2 - 4 } & 0,078256 & & 0,051719 \\
\hline 32. Monitoramento da satisfação de clientes e partes interessadas & 0,011873 & 0,75 & 0,008904 \\
\hline 33. Monitoramento dos processos e produtos & 0,01389 & 0,75 & 0,010418 \\
\hline 34. Monitoramento da conformidade legal & 0,016932 & 0,75 & 0,012699 \\
\hline 35. Auditoria interna & 0,007668 & 0,75 & 0,00575 । \\
\hline 36. Análise de dados & 0,009905 & 0,50 & 0,004952 \\
\hline 37. Controle de não-conformidade & 0,01032 & 0,50 & 0,00516 \\
\hline 38. Gestão das melhorias & 0,007668 & 0,50 & 0,003834 \\
\hline \multicolumn{2}{|c|}{ Desempenho geral da organização B } & & $\mathbf{5 9 , 5 4 \%}$ \\
\hline
\end{tabular}

Observou-se que para esta organização o critério mais importante para o seu desempenho é o sistema de gestão, com um bom nível de desempenho, podendo ainda ser melhorado. Tal fato é devido à implantação do sistema de gestão da qualidade ter sido implementado há mais dez anos, como na Organização A.

Dentro do critério de sistema de gestão a Organização $B$ destaca como sendo mais importante para o seu desempenho o subcritério controle de documentos, obtendo um nível de desempenho maior dentre os outros subcritérios avaliados.

O critério gestão do ciclo de vida do produto apresentou o menor índice nesta avaliação de desempenho, seguido do critério processos para a segurança e saúde ocupacional.Tal fato é, segundo a equipe responsável pelo sistema de gestão, devido a organização estar iniciando a implantação do sistema de gestão ambienta e de segurança e saúde ocupacional, visando a sua integração com os sistemas de gestão da qualidade já implantado.

O desempenho geral da Organização B foi de $59,54 \%$, sendo um valor acima da média da escala de desempenho. Este desempenho reflete a necessidade da implementação dos sistemas de ambiental e de segurança e saúde ocupacional, visando a melhoria do desempenho da organização como um todo.

\section{Conclusões}

Os resultados apresentados demonstraram que 0 método proposto é uma ferramenta eficiente para diagnosticar de forma simples e flexível o desempenho de uma organização que implantou ou está implantando um sistema de gestão da qualidade, meio ambiente e segurança e saúde ocupacional com objetivo de melhoria do desempenho dos seus processos internos produtivos ou de apoio administrativo.

A eficiência do método proposto pôde ser observada no decorrer de sua aplicação, através das seguintes caraterísticas:

- Grande envolvimeto das pessoas que participaram da aplicação do método proposto para avaliação do sistema de gestão;

- Facilidade na compreenção e interpretação dos requisitos avaliados pelo método proposto;

- Facilidade de análise dos resultados e tomada de decisão por parte dos membros da diretoria e das gerências das organizações envolvidas.

As duas organizações apresentaram níveis de desempenho similares sendo que cada organização tem sua peculiaridade, seus pontos fortes individuais e oportunidades de melhorias que cada uma deve trabalhar para buscar a melhoria dos seus processos produtivos.

Foram estabelecidos os elementos característicos e procedimentos de avaliação com base no Anexo A da norma ISO 9004:2000, no método AHP e da fundamentação teórica que deu suporte ao contexto deste trabalho. 
Identificaram-se os elementos característicos de procedimentos de avaliação em outras áreas de estudo a partir da observação de aspectos e experiências realizados, a partir da elaboração e aplicação, em organizações, do método proposto;

Foram definidos os critérios, itens e requisitos relevantes que permitiram avaliar as organizações que participaram deste trabalho frente ao seu desempenho servindo de suporte à tomada de decisões estratégicas que possam elevar estas organizações a um patamar de excelência nos seus respectivos meios de atuação.

\section{Referências}

ABNT NBR ISO | 400 I, Sistemas de gestão ambiental Requisitos. Dez. 2004

ABNT NBR ISO 900 I, Sistemas de gestão da qualidade Requisitos. Dez. 2000.

ABNT NBR ISO 9004, Sistemas de gestão da qualidade Diretrizes para melhorias de desempenho. Dez. 2000.

CERQUERA, J. P. Sistemas de gestão integrados: ISO 900I, ISO I400।, NBR I600 I, OHSAS I800I, SA 8000: Conceitos e aplicações. - Rio de Janeiro: Qualitymark, 2006.

CORRÊAA.A.A. Avaliação de um sistema integrado de gestão: um estudo na indústria automotiva. Dissertação (Mestrado em Engenharia). UFRS, Porto Alegre, RS. 2004.

DE CICCO, F. OHSAS I800। - Especificação para sistemas de gestão da segurança e saúde no trabalho - QSP $-2003$.

DE CICCO, F. SIGs - Sistemas integrados de gestão. Da teoria à prática-QSP, 2003.

FORMAN, E.; Selly, M. Decisions by objectives. Expert Choice, Inc.(200I). Disponível em <http://www.expertchoice.com/service/books/dbo.html>, acesso em desembro de 2008.

GARCIA-MIRANDA, C. E.; Muñiz, B. F.; Sánchez, A. S. Organizacón de la preventiva y gestión de la seguridad y salud laboral en la minería española: experience de las empresas certificadas ISO 900 I. Revista Dirección y Organización, Num. 40, pp. 86-98.

GOMES, L. F. A. M.; et al.Tomada de decisões em cenários complexos: introdução aos métodos discretos do apoio multicritério à decisão. São Paulo. Ed. Thomson Learning, 2004.

JORGENSEN, T. H.; Remmen, A.; Mellado, M. D. Integrated management systems - three diferrent levels of integration. - Journal of Cleaner Prodction. 2006.

KARAPETROVIC, S. Strategies for the integration of management systems and standards. - The TQM Magazine. Vol. I4, n. ${ }^{\circ}$ I, pp. 6I-67. 2002.

LUZ, S. O. C.; Sellitto, M; Gomes, L. P. Medição de desempenho ambiental baseada em método multicriterial de apoio à decisão: Estudo de caso na indústria automotiva. Revista Gestão e Produção, v. I 3, n3, p. 557-570, set.dez. 2006.

SAATY,T. L. Analytic Hierarchy Process, vol. 2 Decision Making for Leaders. Vol. Il of the AHP Series Thomas L. Saaty, 3 I 5 pp., RWS Publ. (new ed.), 200 I.

SAATY,T. L. Relative Measurement and Its Generalization in Decision Making. Why Pairwise Comparisons are Central in Mathematics for the Measurement of Intangible Factors. The Analytic Hierarchy/Network Process. Rev. R. Acad. Cien. Serie A. Mat.VOL. 102 (2), 2008, pp. 25।-3I 8.

SALOMON,V.A. P. Desempenho da Modelagem do Auxílio à Decisão por Múltiplos Critérios na Análise do Planejamento e Controle da Produção. Tese (Doutorado em Engenharia), Poli/USP, São Paulo, SP, 2004.

SHIMIZU, T. Decisão nas Organizações: introdução aos problemas de decisão encontrados nas organizações e nos sistemas de apoio à decisão. São Paulo. Ed. Atlas. 2006.

VELASQUEZ,Y. N.; Nuñez, M.; Monroy, C. R. Aplicación de la técnica AHP para evaluar el efecto de los valores organizacionales en la productividad. Revista Dirección y Organización, Num. 4I, pp. 58-67.

WILKINSON, G. and Dale, B. G. Integrated management system: an examination of the concept and theory. The TQM Magazine.Vol. II, n. ${ }^{\circ}$ 2, pp. $95-104.1999$. 


\section{ANEXO I \\ FORMULÁRIO PARA AVALIAÇÃO DO SIG}

Responda marcando com um " $X$ ", com ajuda das orientações descritas no quadro abaixo, o nível de desempenho que a sua organização se encontra.

\begin{tabular}{|c|l|l|}
\hline $\begin{array}{c}\text { NIVEL DE } \\
\text { MATURIDADE }\end{array}$ & NIVEL DE DESEMPENHO & \multicolumn{1}{|c|}{ ORIENTAÇÕES } \\
\hline 1 & Nenhuma abordagem formal & $\begin{array}{l}\text { Não existe procedimento escrito. Nenhum resultado de melhoria. Falta de } \\
\text { treinamento. }\end{array}$ \\
\hline 2 & Abordagem reativa & $\begin{array}{l}\text { Existem procedimentos escritos, porém confusos. Treinamento inadequado. Sistema } \\
\text { baseado em correção de problemas. }\end{array}$ \\
\hline 3 & $\begin{array}{l}\text { Abordagem estável e formal } \\
\text { do sistema }\end{array}$ & $\begin{array}{l}\text { Existem procedimentos escritos com clareza. Treinamento adequado. Estágio inicial } \\
\text { de melhorias no sistema. }\end{array}$ \\
\hline 4 & Ênfase em melhoria continua & $\begin{array}{l}\text { Processos de melhoria em uso, desenvolvimento de métodos e utilização de } \\
\text { ferramentas para melhoria continua. }\end{array}$ \\
\hline 5 & $\begin{array}{l}\text { Desempenho melhor da } \\
\text { classe }\end{array}$ & $\begin{array}{l}\text { Processo de melhoria fortemente integrado; resultados de melhor da classe quando } \\
\text { comparado com referências de excelência. }\end{array}$ \\
\hline
\end{tabular}

\begin{tabular}{|l|c|c|c|c|}
\hline \multirow{2}{*}{ QUESTÕES: Sistema de Gestão } & \multicolumn{3}{|c|}{ NIVEIS DE } \\
DESEMPENHO \\
\cline { 3 - 5 } & $\mathbf{1}$ & $\mathbf{2}$ & $\mathbf{3}$ & $\mathbf{5}$ \\
\hline $\begin{array}{l}\text { 1. Como a organização estrutura a documentação de forma a promover uma operação eficaz e eficiente de seus } \\
\text { processos? }\end{array}$ & & & \\
\hline 2. Como são utilizados os documentos para apoiar a operação eficaz e eficiente dos processos da organização? & & & & \\
\hline 3. Como são utilizados os registros para apoiar a operação eficaz e eficiente dos processos da organização? & & & \\
\hline
\end{tabular}

\section{QUESTÕES: Responsabilidade da Direção}

4. Como a Alta Direção demonstra sua liderança, comprometimento e envolvimento?

5. Como a organização identifica forma continua as necessidades e expectativas do cliente?

6. Como a política do sistema integrado de gestão é formulada de modo a assegurar que as necessidades e expectativas de todas as partes interessadas são entendidas?

7. Como os objetivos e as metas são planejados e desdobrados para cada nivel gerencial para assegurar a contribuição individual ao seu alcance?

8. Como a Alta Direção assegura que as responsabilidades estão estabelecidas e comunicadas para as pessoas na organização.

\section{QUESTÕES: Gestão de Recursos}

9. Como a direção planeja, fornece, controla e monitora os recursos financeiros necessários para manter um sistema de gestão integrado eficaz e eficiente e para assegurar que se atinjam os objetivos da organizacão?

10. Como a Alta Direção promove o envolvimento e o apoio de pessoas para a melhoria da eficácia e eficiência da organização?

11. Como a direção assegura que a infra-estrutura é apropriada para atingir os objetivos da organização?

12. Como a direção assegura que as informações apropriadas estão facilmente disponiveis para tomada de decisão baseada em fatos?

13. Como a direção envolve fornecedores na identificação das necessidades de aquisição e no desenvolvimento de estratégias conjuntas?

\begin{tabular}{|c|c|c|c|c|c|}
\hline & \multicolumn{5}{|c|}{ NIVEIS DE } \\
\multicolumn{4}{|c|}{ DESEMPENHO } \\
\hline & 1 & 2 & 3 & 4 & 5 \\
\hline & & & & & \\
\hline & & & & & \\
\hline e & & & & & \\
\hline a & & & & & \\
\hline as & & & & & \\
\hline
\end{tabular}

\begin{tabular}{|c|c|c|c|c|c|}
\hline \multirow[t]{2}{*}{ QUESTÕES: Realização do Produto } & \multicolumn{5}{|c|}{$\begin{array}{c}\text { NIVEIS DE } \\
\text { DESEMPENHO }\end{array}$} \\
\hline & & 2 & 3 & 4 & \\
\hline 14. Como é planejada a realização dos produtos, considerando a satisfação dos clientes? & & & & & \\
\hline $\begin{array}{l}\text { 15. Como a direção definiu os processos relacionados aos clientes e a outras partes interessadas para assegurar } \\
\text { a consideração das necessidades e expectativas dessas partes? }\end{array}$ & & & & & \\
\hline $\begin{array}{l}\text { 16. Como são administrados na prática os processos de projeto e de desenvolvimento, incluindo a definição de } \\
\text { requisitos de projeto e de desenvolvimento e a obtenção dos resultados planejados? }\end{array}$ & & & & & \\
\hline $\begin{array}{l}\text { 17. Como os processos de realização do produto são gerenciados desde as entradas até as saídas, } \\
\text { considerando atividades tais como verificação e validação? }\end{array}$ & & & & & \\
\hline
\end{tabular}




\section{ANEXO I \\ FORMULÁRIO PARA AVALIAÇÃO DO SIG (continuación)}

18. Como são gerenciados os processos de aquisição de bens e serviços desde a especificação até sua aceitação?

19. Como a direção controle os dispositivos de medição e monitoramento para assegurar que dados corretos estão sendo obtidos e utilizados?

\section{QUESTÕES: Processos para a segurança e saúde ocupacional}

20. Como a organização identifica os perigos, controla e avalia os riscos existentes para a segurança do trabalhador?

21. Como a organização identifica os perigos, controla e avalia os riscos existentes para a saúde do trabalhador?

22. Como a direção assegura que 0 ambiente de trabalho promove motivação, satisfação, desenvolvimento melhor desempenho das pessoas na organização?

23. Como a organização adequou as inovações dos seus processos produtivos face à saúde e segurança dos seus funcionários?

24. Como a organização incorporou na sua estratégia para o sistema integrado de gestão, planos de ação para assegurar, que quando novas instalações, máquinas, equipamentos e materiais forem introduzidos nos locais de trabalho, os perigos identificados e os riscos avaliados e controlados?

25. Como a organização estabeleceu metodologias para que sejam observadas práticas seguras de trabalho, a fim de parar, bloquear e reiniciar os equipamentos antes e após a conclusão dos trabalhos de manutenção?

26. Como a organização implementou, atualiza e mantém seu plano de prontidão para atendimento a emergências compativel com a natureza de suas atividades?

27. Como a organização investiga seus acidentes e incidentes e avalia sua causalidade?
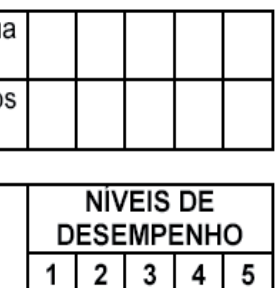

\section{QUESTÕES: Gestão do ciclo de vida do produto}

28. Como a organização assegura a disponibilidade dos recursos naturais e energia necessária para seus processos de realização, de forma a não comprometer o meio ambiente?

29. Como a organização busca adequar seus produtos a uma rotulagem verde e usa estas informações para melhoria do seu desempenho?

30. Como a organização com a colaboração de seus funcionários, procura integrar o desempenho sustentável à racionalização do processo produtivo?

31. Como a organização faz uso dos conhecimentos técnicos para trabalhar a reciclagem, a reutilização, a redução, a armazenagem, o transporte e o tratamento de resíduos para a melhoria dos seus processos produtivos?

\begin{tabular}{|c|c|c|c|c|c|}
\hline \multirow[t]{2}{*}{ QUESTÕES: Medição, análise e melhoria } & \multicolumn{5}{|c|}{$\begin{array}{c}\text { NIVEIS DE } \\
\text { DESEMPENHO }\end{array}$} \\
\hline & 1 & 2 & 3 & 4 & 5 \\
\hline $\begin{array}{l}\text { 32. Como a direção promove a importância das atividades de medição, análise e melhoria para assegurar que o } \\
\text { desempenho da organização resulta em satisfação do cliente e das partes interessadas? }\end{array}$ & & & & & \\
\hline 33. Como a organização monitora seus processos e a qualidade de seus produtos? & & & & & \\
\hline 34. Como a organização monitora o atendimento aos requisitos legais? & & & & & \\
\hline $\begin{array}{l}\text { 35. Como a organização avalia os resultados de auditorias internas e externas, de forma a obter melhorias no seu } \\
\text { sistema integrado de gestão? }\end{array}$ & & & & & \\
\hline 36. Como a organização analisa os dados para avaliar seu desempenho e identificar áreas de melhoria? & & & & & \\
\hline $\begin{array}{l}\text { 37. Como a organização controla e analisa as não-conformidades para a sua aprendizagem e melhoria de seu } \\
\text { desempenho? }\end{array}$ & & & & & \\
\hline $\begin{array}{l}\text { 38. Como a direção assegura o uso de métodos sistemáticos, ferramentas, as ações corretivas e ações } \\
\text { preventivas para melhorar o desempenho da organizacão? }\end{array}$ & & & & & \\
\hline
\end{tabular}

SUGESTÕES E COMENTÁROS SOBRE O QUESTIONÁRIO: (se necessário utilize o verso) 